\title{
MEAN VALUES OF DIVISORS TWISTED BY QUADRATIC CHARACTERS
}

\author{
PENG GAO
}

\begin{abstract}
In this paper, we evaluate the sum $\sum_{m, n}\left(\frac{m}{n}\right) d(n)$, where $\left(\frac{m}{n}\right)$ is the Kronecker symbol and $d(n)$ is the divisor function.
\end{abstract}

\section{Mathematics Subject Classification (2010): 11N37, 11L05, 11L40}

Keywords: divisor function, mean value, quadratic Dirichlet character

\section{INTRODUCTION}

As estimations for character sums have wide applications in analytic number theory, many important results are obtained in this direction. Among the many characters studied in the literature, quadratic Dirichlet characters receive more attentions due to their relations to ranks of elliptic curves, class numbers, etc. A special case of the well-known Pólya-Vinogradov inequality (see [3, Chap. 23]) asserts that for any positive $m \neq \square$ (where $\square$ represents the square of a rational integer), and any $Y>0$,

$$
\sum_{n \leq Y}\left(\frac{m}{n}\right) \ll m^{1 / 2} \log m
$$

where $\left(\frac{m}{.}\right)$ is the Kronecker symbol.

One may regard the above Pólya-Vinogradov inequality as a first moment estimation for quadratic Dirichlet characters. In view of this, it is natural to ask for similar estimations for higher moments involving quadratic character sums. For the second moment, we note the following mean square estimate for quadratic Dirichlet characters due to M. V. Armon [1, Theorem 1]:

$$
\sum_{\substack{|D| \leq X \\ D \in \mathcal{D}}}\left|\sum_{n \leq Y}\left(\frac{D}{n}\right)\right|^{2} \ll X Y \log X,
$$

where $\mathcal{D}$ is the set of non-square quadratic discriminants. Weaker estimations were obtained earlier by M. Jutila [6,7] to study problems related to the mean values of class numbers of quadratic imaginary fields and the second moment of Dirichlet $L$-functions with primitive quadratic characters.

As the Pólya-Vinogradov inequality demonstrates certain square root cancellation, it is not possible to obtain an asymptotic expression for the character sum given in the left-hand side of (1.1). However, things change if we consider an extra average over $m \leq X$ as well. In fact, it is relatively easy to obtain such an asymptotic formula when $X, Y$ are far apart in size, as the Pólya-Vinogradov inequality (1.1) itself allows us to have a good control on the error term. On the other hand, things become much subtler when $X$ and $Y$ are of comparable size and it is not until in year 2000 that J. B. Conrey, D. W. Farmer, and K. Soundararajan [2] determined completely the asymptotic formula for all $X, Y>0$ of the following sum:

$$
\sum_{\substack{m \leq Y \\(m, 2)=1}} \sum_{\substack{n \leq Y \\(n, 2)=1}}\left(\frac{m}{n}\right) .
$$

The above expression motivates us to consider similar sums involving quadratic Dirichlet characters. One can certainly do so by considering mean values of any arithmetic function twisted by quadratic characters. We shall, however, in this paper, consider the following more concrete sum:

$$
S(X, Y)=\sum_{\substack{m \leq X \\(m, 2)=1}} \sum_{\substack{n \leq Y \\(n, 2)=1}}\left(\frac{m}{n}\right) d(n) .
$$

Date: April 16, 2019. 
One reason that leads to our consideration of the above sum is that, when we square out the sums in (1.2) and interchange the order of summations, we find that

$$
\sum_{\substack{D \mid \leq X \\ D \in \mathcal{D}}}\left|\sum_{n \leq Y}\left(\frac{D}{n}\right)\right|^{2}=\sum_{n \leq Y} \sum_{\substack{D \mid \leq X \\ D \in \mathcal{D}}}\left(\frac{D}{n}\right) d(n)+\sum_{\substack{n>Y \\ n=1 \\ n=1 \\ n_{1} \leq Y, n_{2} \leq Y}} \sum_{\substack{|D| \leq X \\ D \in \mathcal{D}}}\left(\frac{D}{n}\right) .
$$

When one compares the first sum on the right-hand side expression above with $S(X, Y)$, it is easy to see that they differ only by certain restrictions on the sums and this is what gets us into the consideration of $S(X, Y)$.

Another reason for us to consider $S(X, Y)$ comes from the work of K. Soundararajan in [9], in which he evaluated mollified first and second moments of quadratic Dirichlet $L$-functions to show that at least $87.5 \%$ such $L$-functions are non-vanishing at the central point. An important part of the arguments in [9] involves with the evaluation of smoothed sums of the following type:

$$
\sum_{(d, 2)=1} \mu^{2}(d) \sum_{n}\left(\frac{8 d}{n}\right) \frac{d_{j}(n)}{\sqrt{n}} \omega_{j}\left(\frac{n}{d^{j / 2}}\right) F\left(\frac{d}{X}\right), \quad j=1,2 .
$$

Here one may regard $\omega_{j}, j=1,2$ and $F$ as Schwarz class functions and $d_{1}(n)=1, d_{2}(n)=d(n)$ for all $n$. Thus, other than the factor $1 / \sqrt{n}$, the above sums when $j=1$ can be regarded as a smoothed version of the sums considered in (1.3), except that in the inner sum above, we are essentially summing over $n$ going up to the size of $d$, while in (1.3), the lengths of the summations are independent. In the same fashion, $S(X, Y)$ can be regarded as a study of the above sums for $j=2$ by making the lengths of the sums independent.

We are now ready to state our result in the following

Theorem 1.1. For large $X$ and $Y$, we have

$$
\begin{aligned}
S(X, Y)= & \frac{X Y^{1 / 2} \log ^{2} Y}{16} \eta(1)+X Y^{1 / 2} P_{1}(\log Y)+X^{3 / 2}(\log X+2 \gamma) C_{1}\left(\frac{Y}{X}\right)+X^{3 / 2} C_{2}\left(\frac{Y}{X}\right) \\
& +O\left(X^{1+\epsilon} Y^{\frac{1}{4}+\epsilon}\left(\frac{X Y^{1 / 2}+Y X^{1 / 2}}{X Y^{\frac{1}{4}}}\right)^{2 / 3}+X^{1+\epsilon} Y^{1 / 2+\epsilon}\left(\frac{X Y^{\frac{1}{4}}}{X Y^{1 / 2}+Y X^{1 / 2}}\right)^{1 / 3}\left(\frac{Y}{X}\right)^{(1+\epsilon) / 2}\right. \\
& \left.+Y^{3 / 2+\epsilon}\left(\frac{X Y^{\frac{1}{4}}}{X Y^{1 / 2}+Y X^{1 / 2}}\right)^{2 / 3}+X^{1+\epsilon} Y^{\epsilon}\left(\frac{Y}{X}\right)^{2}\left(\frac{X Y^{1 / 2}+Y X^{1 / 2}}{X Y^{\frac{1}{4}}}\right)^{2}\right) .
\end{aligned}
$$

where $\eta(s)$ is given in (3.7), $P_{1}(x)$ is given in (3.10) and $C_{1}(\alpha), C_{2}(\alpha)$ are functions of $\alpha \geq 0$ given in (3.21).

It is easy to see that (1.4) gives a valid asymptotic formula when $X^{\epsilon} \ll Y \ll X^{1-\epsilon}$ for any $\epsilon>0$. We also note that via integration by parts that for $i=1,2$,

$$
C_{i}(\alpha)=O\left(\alpha^{3 / 2} \log \alpha\right), \quad \alpha \rightarrow 0
$$

Unlike the case studied in [2], the behavior of $C_{i}(\alpha)$ when $\alpha \rightarrow \infty$ is more complicated to analyze and we shall not worry about it here.

Our strategy for proving Theorem (1.1) is similar to that in the proof of Theorem 1 in [2]. We first replace $S(X, Y)$ by the following smoothed sum

$$
\mathcal{S}(X, Y)=\sum_{\substack{m \leq X \\(m, 2)=1}} \sum_{\substack{n \leq Y \\(n, 2)=1}}\left(\frac{m}{n}\right) d(n) \Phi\left(\frac{n}{Y}\right) W\left(\frac{m}{X}\right) .
$$

Here $\Phi$ and $W$ are smooth functions supported in $(0,1)$, satisfying $\Phi(t)=W(t)=1$ for $t \in\left(\frac{1}{U}, 1-\frac{1}{U}\right)$ for a parameter $U$, and such that

$$
\Phi^{(j)}(t), W^{(j)}(t) \ll_{j} U^{j}
$$

for all integers $j \geq 0$.

We shall apply a large sieve result for quadratic Dirichlet characters to control the size of $S(X, Y)-\mathcal{S}(X, Y)$. We then apply the Poisson summation to evaluate $\mathcal{S}(X, Y)$. Choosing $U$ optimally leads to the assertion of Theorem 1.1

\section{Preliminaries}

In this section, we gather a few auxiliary results needed in the proof of Theorem 1.1. 
2.1. Gauss sums. For all odd integers $k$ and all integers $m$, we introduce the following Gauss-type sums as in [9, Sect. $2.2]$

$$
G_{m}(k)=\left(\frac{1-i}{2}+\left(\frac{-1}{k}\right) \frac{1+i}{2}\right) \sum_{a(\bmod k)}\left(\frac{a}{k}\right) e\left(\frac{a m}{k}\right),
$$

where $e(x)=e^{2 \pi i x}$. We quote [9, Lemma 2.3] which determines $G_{m}(k)$.

Lemma 2.2. If $\left(k_{1}, k_{2}\right)=1$ then $G_{m}\left(k_{1} k_{2}\right)=G_{m}\left(k_{1}\right) G_{m}\left(k_{2}\right)$. Suppose that $p^{a}$ is the largest power of $p$ dividing $m$ (put $a=\infty$ if $m=0$ ). Then for $b \geq 1$ we have

$$
G_{m}\left(p^{b}\right)=\left\{\begin{array}{cl}
0 & \text { if } b \leq a \text { is odd, } \\
\varphi\left(p^{b}\right) & \text { if } b \leq a \text { is even, } \\
-p^{a} & \text { if } b=a+1 \text { is even, } \\
\left(\frac{m / p^{a}}{p}\right) p^{a} \sqrt{p} & \text { if } b=a+1 \text { is odd, } \\
0 & \text { if } b \geq a+2 .
\end{array}\right.
$$

2.3. Poisson Summation. For a Schwartz function $F$, we define

$$
\widetilde{F}(\xi)=\frac{1+i}{2} \hat{F}(\xi)+\frac{1-i}{2} \hat{F}(-\xi)=\int_{-\infty}^{\infty}(\cos (2 \pi \xi x)+\sin (2 \pi \xi x)) F(x) \mathrm{d} x,
$$

where $\hat{F}$ denotes the Fourier transform of $F$.

We have the following Poisson summation formula from [9, Lemma 2.6]:

Lemma 2.4. Let $W$ be a smooth function with compact support on the positive real numbers. For any odd integer $n$,

$$
\sum_{(d, 2)=1}\left(\frac{d}{n}\right) W\left(\frac{d}{X}\right)=\frac{X}{2 n}\left(\frac{2}{n}\right) \sum_{k}(-1)^{k} G_{k}(n) \widetilde{W}\left(\frac{k X}{2 n}\right),
$$

where $\widetilde{W}$ is defined in (2.2) and $G_{k}(n)$ is defined in (2.1).

We shall apply the above lemma to the function $W$ defined in the Introduction. Here we recall the following estimations on this $W$ from [2, (4.1)]:

$$
\widetilde{W}^{(\mu)}(t) \ll_{j} U^{j-1} t^{-j}
$$

for all integers $\mu \geq 0, j \geq 1$ and all real $t>0$.

On the other hand, we note that it follows from [2, (4.2)] that for the same $W$,

$$
\widetilde{W}(t)=\frac{1-\cos (2 \pi t)+\sin (2 \pi t)}{2 \pi t}+O\left(\frac{1}{U}\right) .
$$

2.5. A large sieve for quadratic Dirichlet characters. Another important tool needed in the proof of Theorem 1.1 is the following large sieve inequality for quartic Dirichlet characters due to D. R. Heath-Brown [4, Theorem 1].

Lemma 2.6. Let $M, N$ be positive integers, and let $\left(a_{n}\right)_{n \in \mathbb{N}}$ be an arbitrary sequence of complex numbers. Then we have

$$
\sum_{m \leq M}^{*}\left|\sum_{n \leq N}^{*} a_{n}\left(\frac{n}{m}\right)\right|^{2} \ll_{\varepsilon}(M+N)(M N)^{\varepsilon} \sum_{n \leq N}\left|a_{n}\right|^{2},
$$

for any $\varepsilon>0$, where the asterisks indicate that $m$ and $n$ run over positive odd square-free integers.

\section{Proof of Theorem 1.1}

3.1. Initial Reductions. Before we evaluate $\mathcal{S}(X, Y)$, we first want to estimate $S(X, Y)-\mathcal{S}(X, Y)$, where $\mathcal{S}(X, Y)$ is defined in (1.5). It is easy to see that $S(X, Y)-\mathcal{S}(X, Y)$ can be expressed as linear combinations of sums of the following forms:

$$
\sum_{\substack{m \in I_{1} \\(m, 2)=1}} \sum_{\substack{n \in I_{2} \\(n, 2)=1}}\left(\frac{m}{n}\right) d(n) H(n, m),
$$

where $H(x, y)=1$ or $\Phi\left(\frac{n}{Y}\right) W\left(\frac{m}{X}\right), I_{1} \in\{[0, X / U],[X(1-1 / U), X],[0, X]\}, I_{2} \in\{[0, Y / U],[Y(1-1 / U), Y],[0, Y]\}$, and the case $I_{1}=[0, X], I_{2}=[0, Y]$ is excluded. 
As the arguments are similar, we only treat the following sum:

$$
\sum_{\substack{m \in[X(1-1 / U), X] \\(m, 2)=1}} \sum_{\substack{n \in[0, Y] \\(n, 2)=1}}\left(\frac{m}{n}\right) d(n) .
$$

We note that

$$
\begin{aligned}
& \sum_{\substack{m \in[X(1-1 / U), X] \\
(m, 2)=1}} \sum_{\substack{n \in[0, Y] \\
(n, 2)=1}}\left(\frac{m}{n}\right) d(n)=\sum_{\substack{a^{2} \leq X \\
(a, 2)=1}} \sum_{\substack{b \in\left[X(1-1 / U) / a^{2}, X / a^{2}\right] \\
(b, 2)=1}} \sum_{\substack{e^{2} \leq Y \\
(e, 2)=1}} \sum_{\substack{f \leq Y / e^{2} \\
(f, 2)=1}} \mu^{2}(b) \mu^{2}(f)\left(\frac{a^{2} b}{e^{2} f}\right) d\left(e^{2} f\right) \\
& =\sum_{\substack{a^{2} \leq X \\
(a, 2)=1}} \sum_{\substack{e^{2} \leq Y \\
(e, 2 a)=1}} \sum_{\substack{b \in\left[X(1-1 / U) / a^{2}, X / a^{2}\right] \\
(b, 2 e)=1}} \sum_{\substack{f \leq Y / e^{2} \\
(f, 2 a)=1}} \mu^{2}(b) \mu^{2}(f)\left(\frac{b}{f}\right) d\left(e^{2} f\right) .
\end{aligned}
$$

For fixed $a, b$, we apply the Cauchy-Schwarz inequality and Lemma 2.6 to see that

$$
\begin{aligned}
& \sum_{\substack{b \in\left[X(1-1 / U) / a^{2}, X / a^{2}\right] \\
(b, 2 e)=1}} \sum_{\substack{f \leq Y / e^{2} \\
(f, 2 a)=1}} \mu^{2}(b) \mu^{2}(f)\left(\frac{b}{f}\right) d\left(e^{2} f\right)
\end{aligned}
$$

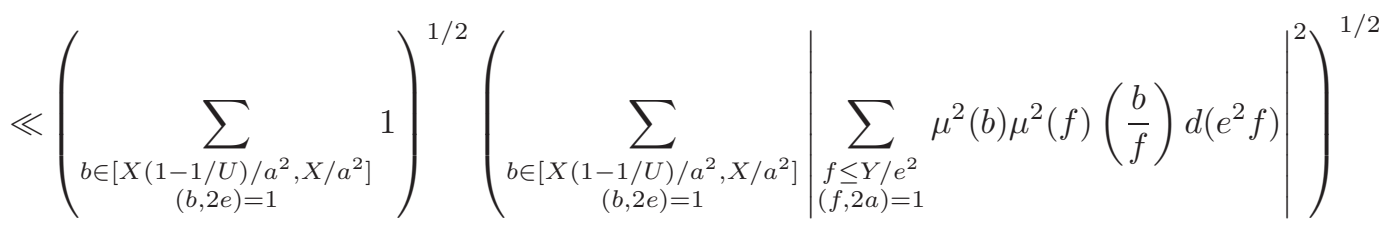

$$
\begin{aligned}
& \ll \sqrt{\frac{X}{U a^{2}}}\left(\left(\frac{X}{a^{2}}+\frac{Y}{e^{2}}\right)(X Y)^{\epsilon} \sum_{\substack{f \leq Y / e^{2} \\
(f, 2 a)=1}} d^{2}\left(e^{2} f\right)\right)^{1 / 2} \ll(X Y)^{\epsilon} \sqrt{\frac{X}{U a^{2}}}\left(\sqrt{\frac{X Y}{a^{2} e^{2}}}+\frac{Y}{e^{2}}\right) .
\end{aligned}
$$

Applying the above estimation to the right-hand side of (3.2), we see upon summing over $a$ and $e$ that

$$
\sum_{\substack{m \in[X(1-1 / U), X] \\(m, 2)=1}} \sum_{\substack{n \in[0, Y] \\(n, 2)=1}}\left(\frac{m}{n}\right) d(n) \ll(X Y)^{\epsilon} \frac{X Y^{1 / 2}+Y X^{1 / 2}}{\sqrt{U}} .
$$

One checks that the above estimation applies to all other types of sums given in (3.1). We then conclude that

$$
S(X, Y)-\mathcal{S}(X, Y) \ll(X Y)^{\epsilon} \frac{X Y^{1 / 2}+Y X^{1 / 2}}{\sqrt{U}} .
$$

Next, we treat $\mathcal{S}(X, Y)$ by applying Lemma 2.4, we see that

$$
\begin{aligned}
\mathcal{S}(X, Y) & =X \sum_{n} \frac{d(n)}{2 n}\left(\frac{2}{n}\right) \Phi\left(\frac{n}{Y}\right) \sum_{k}(-1)^{k} G_{k}(n) \widetilde{W}\left(\frac{k X}{2 n}\right) \\
& =\frac{X \widetilde{W}(0)}{2} \sum_{n}\left(\frac{2}{n}\right) \frac{G_{0}(n) d(n)}{n} \Phi\left(\frac{n}{Y}\right)+\frac{X}{2} \sum_{k \neq 0}(-1)^{k} \sum_{n}\left(\frac{2}{n}\right) \frac{G_{k}(n) d(n)}{n} \Phi\left(\frac{n}{Y}\right) \widetilde{W}\left(\frac{k X}{2 n}\right) \\
& :=M_{0}+M^{\prime} .
\end{aligned}
$$

3.2. The Term $M_{0}$. We estimate $M_{0}$ first. It follows straight from the definition that $G_{0}(n)=\varphi(n)$ if $n=\square$ and $G_{0}(n)=0$ otherwise. Thus

$$
M_{0}=\frac{X \widetilde{W}(0)}{2} \sum_{\substack{(n, 2)=1 \\ n=\square}} \frac{\varphi(n) d(n)}{n} \Phi\left(\frac{n}{Y}\right)
$$

By Mellin inversion, we have

$$
\Phi\left(\frac{n}{Y}\right)=\frac{1}{2 \pi i} \int_{(2)}\left(\frac{Y}{n}\right)^{s} \widehat{\Phi}(s) \mathrm{d} s,
$$


where

$$
\widehat{\Phi}(s)=\int_{0}^{\infty} \Phi(t) t^{s-1} \mathrm{~d} t .
$$

Integration by parts and using (1.6) shows that $\widehat{\Phi}(s)$ is a function satisfying the bound for all $\Re(s)>0$, and integers $D \geq 1$

$$
\widehat{\Phi}(s) \ll(1+|s|)^{-D} U^{D-1} .
$$

We then deduce that

$$
M_{0}=\frac{X \widetilde{W}(0)}{2} \frac{1}{2 \pi i} \int_{(2)} Y^{s} \widehat{\Phi}(s)\left(\sum_{\substack{(n, 2)=1 \\ n=\square}} \frac{\varphi(n) d(n)}{n^{1+s}}\right) \mathrm{d} s=\frac{X \widetilde{W}(0)}{2} \frac{1}{2 \pi i} \int_{(2)} Y^{s} \widehat{\Phi}(s)\left(\sum_{(n, 2)=1} \frac{\varphi\left(n^{2}\right) d\left(n^{2}\right)}{n^{2+2 s}}\right) \mathrm{d} s .
$$

We deduce by comparing the Euler factors that

$$
\sum_{\substack{(n, 2)=1 \\ n=\square}} \frac{\varphi(n) d(n)}{n^{1+s / 2}}=\sum_{(n, 2)=1} \frac{\varphi\left(n^{2}\right) d\left(n^{2}\right)}{n^{2+s}}=\zeta^{3}(s) \eta(s),
$$

where $\eta(s)=\prod_{p} \eta_{p}(s)$ with

$$
\eta_{2}(s)=\left(1-2^{-s}\right)^{3}
$$

and for $p>2$,

$$
\eta_{p}(s)=1-\frac{3}{p^{1+s}}+\left(3-4\left(1-\frac{1}{p}\right)\right) \frac{1}{p^{2 s}}-\frac{1}{p^{1+3 s}} .
$$

From this we see that $\eta(s)$ is absolutely convergent in $\Re(s)>1 / 2$.

We then derive from (3.6) that

$$
M_{0}=\frac{X \widetilde{W}(0)}{2} \frac{1}{2 \pi i} \int Y^{s} \widehat{\Phi}(s) \zeta^{3}(2 s) \eta(2 s) \mathrm{d} s .
$$

We now shift the line of integration in the above expression to the $\Re(s)=1 / 4+\epsilon$ line. We encounter a pole of order 3 at $s=1 / 2$. Thus we may write $M_{0}=M_{0,1}+R_{0}$, where

$$
M_{0,1}=\frac{X \widetilde{W}(0)}{2} \operatorname{Res}_{s=1 / 2} Y^{s} \widehat{\Phi}(s) \zeta^{3}(2 s) \eta(2 s) \text { and } R_{1}=\frac{X \widetilde{W}(0)}{2} \frac{1}{2 \pi i} \int_{\left(\frac{1}{4}+\varepsilon\right)} Y^{s} \widehat{\Phi}(s) \zeta^{3}(2 s) \eta(2 s) \mathrm{d} s .
$$

To estimate $R_{1}$, we note that (see [5, Exercise 3, p. 100]) when $\Re(s)=1 / 4+\epsilon$,

$$
\zeta(2 s) \ll(1+|s|)^{1 / 4+\epsilon} .
$$

Using this to together with (3.5) by taking $D=2$, we deduce that

$$
R_{1} \ll X Y^{\frac{1}{4}+\epsilon} U .
$$

To evaluate $M_{0,1}$, we note the Laurent series expansion for $\zeta(s)$ at $s=1$ (see [8, Corollary 1.16])

$$
\zeta(s)=\frac{1}{s-1}+\gamma+O(s-1)
$$

where $\gamma$ is Euler's constant.

We deduce from (3.9) the following Laurent series expansions at $s=1 / 2$,

$$
\begin{aligned}
\zeta^{3}(2 s) & =\frac{1}{(2 s-1)^{3}}+\frac{3 \gamma}{(2 s-1)^{2}}+\frac{c_{1}}{(2 s-1)}+\ldots, \\
\eta(2 s) & =\eta(1)+2 \eta^{\prime}(1)(s-1 / 2)+2 \eta^{\prime \prime}(1)(s-1 / 2)^{2}+\ldots, \\
Y^{s} & =Y^{1 / 2}+Y^{1 / 2} \log Y(s-1 / 2)+\frac{Y^{1 / 2} \log ^{2} Y}{2}(s-1 / 2)^{2}+\ldots, \\
\widehat{\Phi}(s) & =\widehat{\Phi}(1 / 2)+\widehat{\Phi}^{\prime}(1 / 2)(s-1 / 2)+\frac{\widehat{\Phi}^{\prime \prime}(1 / 2)}{2}(s-1 / 2)^{2}+\ldots,
\end{aligned}
$$

where $c_{1}$ is an absolute constant. 
We further deduce from (3.4) that for some absolute constants $d_{1}, d_{2}$,

$$
\widehat{\Phi}(1 / 2)=2+O\left(\frac{1}{U}\right), \quad \widehat{\Phi}^{\prime}(1 / 2)=d_{1}+O\left(\frac{1}{U}\right), \quad \widehat{\Phi}^{\prime \prime}(1 / 2)=d_{2}+O\left(\frac{1}{U}\right) .
$$

It follows that the residue in $M_{0,1}$ can be written as

$$
\frac{Y^{1 / 2} \log ^{2} Y}{8} \eta(1)+Y^{1 / 2} P_{1}(\log Y)+O\left(\frac{Y^{1 / 2} \log ^{2} Y}{U}\right),
$$

where $P_{1}$ is a polynomial of degree 1 , whose coefficients involve absolute constants $c_{1}, d_{1}, d_{2}$.

We therefore deduce from (2.4) that

$$
M_{0,1}=\frac{X Y^{1 / 2} \log ^{2} Y}{16} \eta(1)+X Y^{1 / 2} P_{1}(\log Y)+O\left(\frac{X Y^{1 / 2} \log ^{2} Y}{U}\right) .
$$

Combining the above estimation with (3.8), we conclude that

$$
M_{0}=\frac{X Y^{1 / 2} \log ^{2} Y}{16} \eta(1)+X Y^{1 / 2} P_{1}(\log Y)+O\left(\frac{X Y^{1 / 2} \log ^{2} Y}{U}+X Y^{\frac{1}{4}+\epsilon} U\right) .
$$

3.3. The Term $M^{\prime}$. Now suppose $k \neq 0$. By Mellin inversion, we have

$$
\Phi\left(\frac{n}{Y}\right) \widetilde{W}\left(\frac{k X}{2 n}\right)=\frac{1}{2 \pi i} \int\left(\frac{Y}{n}\right)^{s} \tilde{f}(s, k) \mathrm{d} s,
$$

where

$$
\tilde{f}(s, k)=\int_{0}^{\infty} \Phi(t) \widetilde{W}\left(\frac{k X}{2 Y t}\right) t^{s-1} \mathrm{~d} t .
$$

Integration by parts and using (2.3) shows that $\tilde{f}(s)$ is a function satisfying the bound for all $\Re(s)>0$, and integers $D, E>0$,

$$
\tilde{f}(s, k) \ll(1+|s|)^{-D}\left(1+\frac{|k| X}{Y}\right)^{-E+D} U^{E-1} .
$$

We apply (3.12) to recast $M^{\prime}$ as

$$
M^{\prime}=\frac{X}{2} \sum_{k \neq 0}(-1)^{k} \int_{(2)} \tilde{f}(s, k) Y^{s} G(1+s, k) \mathrm{d} s .
$$

where

$$
G(1+s, k)=\sum_{n}\left(\frac{2}{n}\right) \frac{G_{k}(n) d(n)}{n^{1+s}}=\sum_{(n, 2)=1} \frac{G_{2 k}(n) d(n)}{n^{1+s}},
$$

where the last equality above follows from the observation that, by changing variables in (2.1), we have $\left(\frac{2}{n}\right) G_{k}(n)=$ $G_{2 k}(n)$ since $n$ is odd.

Write $2 k=k_{1} k_{2}^{2}$ where $k_{1}$ is square-free (possibly $k_{1}=1$ is the trivial character), and $k_{2}$ is positive. In the region $\Re(s)>1$, we recast $G(1+s, k)$ as

$$
G(1+s, k)=L\left(\frac{1}{2}+s, \chi_{k_{1}}\right)^{2} \prod_{p} G_{p}(s, k):=L\left(\frac{1}{2}+s, \chi_{k_{1}}\right)^{2} \mathcal{G}(s, k),
$$

where we denote $\chi_{k}$ for the $\operatorname{Kronecker} \operatorname{symbol}\left(\frac{k}{*}\right)$ and $G_{p}(s, k)$ is defined as follows:

$$
\begin{aligned}
& G_{p}(s, k)=\left(1-\frac{1}{p^{1 / 2+s}}\left(\frac{k_{1}}{p}\right)\right)^{2}, \quad \text { if } p=2, \\
& G_{p}(s, k)=\left(1-\frac{1}{p^{1 / 2+s}}\left(\frac{k_{1}}{p}\right)\right)^{2} \sum_{r=0}^{\infty} \frac{d\left(p^{r}\right)}{p^{r(1 / 2+s)}} \frac{G_{2 k}\left(p^{r}\right)}{p^{r / 2}}, \quad \text { if } p \neq 2 .
\end{aligned}
$$


By Lemma 2.2, we see that for a generic $p \nmid 2 k, G_{p}(s, k)=1-\frac{3}{p^{1+2 s}}+\frac{2}{p^{3 / 2+3 s}}\left(\frac{k_{1}}{p}\right)$, this shows that $\mathcal{G}(s, k)$ is holomorphic in $\Re(s)>0$. From our evaluation of $G_{p}(s, k)$ for $p \nmid 2 k$ we see that for $\Re s \geq \epsilon$,

$$
\mathcal{G}(s, k) \ll|k|^{\epsilon} \prod_{p \mid 2 k}\left|G_{p}(s, k)\right| .
$$

To derive a bound for $G_{p}(s, k)$ when $p \mid 2 k$, we suppose that $p^{a} \| 2 k$. By the trivial bound $\left|G_{k}\left(p^{r}\right)\right| \leq p^{r}$, it follows that $\left|G_{p}(s, k)\right| \leq(a+1)^{2}$ for these $p$. We then conclude that for $\Re s \geq \epsilon$,

$$
\mathcal{G}(s, k) \ll|k|^{\epsilon} .
$$

Using this, we see that

$$
M^{\prime}=\frac{X}{2} \sum_{k \neq 0}(-1)^{k} \int_{(2)} \tilde{f}(s, k) Y^{s} L\left(\frac{1}{2}+s, \chi_{k_{1}}\right)^{2} \mathcal{G}(s, k) \mathrm{d} s .
$$

We now move the line of integration to the line $\Re(s)=\epsilon$ to see that we encounter poles only when $k_{1}=1$ (so that $\left.L\left(s, \chi_{k_{1}}\right)=\zeta(s)\right)$ at $s=1 / 2$, in which case there is a pole of order 2 at $s=1 / 2$. Thus we may write $M_{2}^{\prime}=M_{1,2}+R_{2}$, where (by an obvious change of notation, writing $2 k^{2}$ in place of the corresponding $k$ and observing that $k_{1}^{2}=k_{2}^{2}$ if and only if $k_{1}= \pm k_{2}$ )

$$
M_{1,2}=X \operatorname{Res}_{s=1 / 2} \sum_{k=1}^{\infty} Y^{s} \tilde{f}\left(\frac{1}{2}, 2 k^{2}\right) \mathcal{G}\left(\frac{1}{2}, 2 k^{2}\right) \zeta\left(\frac{1}{2}+s\right)^{2}
$$

and

$$
R_{2}=\frac{X}{2} \sum_{k \neq 0}(-1)^{k} \frac{1}{2 \pi i} \int_{(\varepsilon)} \tilde{f}(s, k) Y^{s} L\left(\frac{1}{2}+s, \chi_{k_{1}}\right)^{2} \mathcal{G}(s, k) \mathrm{d} s .
$$

To evaluate $M_{1,2}$, we note that it follows from (3.9) that near $s=1 / 2$,

$$
\zeta(1 / 2+s)^{2}=\frac{1}{(s-1 / 2)^{2}}+\frac{2 \gamma}{s-1 / 2}+O(s-1 / 2) .
$$

It follows that

$$
\begin{aligned}
M_{1,2}= & X Y^{1 / 2} \log Y \sum_{k=1}^{\infty} \tilde{f}\left(\frac{1}{2}, 2 k^{2}\right) \mathcal{G}\left(\frac{1}{2}, 2 k^{2}\right) \\
& +X Y^{1 / 2} \sum_{k=1}^{\infty} \tilde{f}\left(\frac{1}{2}, 2 k^{2}\right) \mathcal{G}\left(\frac{1}{2}, 2 k^{2}\right)\left(2 \gamma+\frac{\tilde{f}^{\prime}\left(\frac{1}{2}, 2 k^{2}\right)}{\tilde{f}\left(\frac{1}{2}, 2 k^{2}\right)}+\frac{\mathcal{G}^{\prime}\left(\frac{1}{2}, 2 k^{2}\right)}{\mathcal{G}\left(\frac{1}{2}, 2 k^{2}\right)}\right) .
\end{aligned}
$$

We further note that when $s=1 / 2$, we have by Lemma 2.2 that

$$
\sum_{r=0}^{\infty} \frac{d\left(p^{r}\right)}{p^{r(1 / 2+s)}} \frac{G_{4 k^{2}}\left(p^{r}\right)}{p^{r / 2}}>1 .
$$

We deduce from this easily that

$$
\frac{\mathcal{G}^{\prime}\left(\frac{1}{2}, 2 k^{2}\right)}{\mathcal{G}\left(\frac{1}{2}, 2 k^{2}\right)} \ll|k|^{\epsilon} .
$$

We apply the definition of $\tilde{f}(s, k)$ given in (3.13) together with the bound (3.15) to see that

$$
\begin{aligned}
\sum_{k=1}^{\infty} \tilde{f}\left(\frac{1}{2}, 2 k^{2}\right) \mathcal{G}\left(\frac{1}{2}, 2 k^{2}\right) & =\int_{0}^{\infty} \frac{\Phi(t)}{\sqrt{t}} \sum_{k=1}^{\infty} \widetilde{W}\left(\frac{k^{2} X}{Y t}\right) \mathcal{G}\left(\frac{1}{2}, 2 k^{2}\right) \mathrm{d} t \\
\sum_{k=1}^{\infty} \tilde{f}^{\prime}\left(\frac{1}{2}, 2 k^{2}\right) \mathcal{G}\left(\frac{1}{2}, 2 k^{2}\right) & =\int_{0}^{\infty} \frac{\Phi(t) \log t}{\sqrt{t}} \sum_{k=1}^{\infty} \widetilde{W}\left(\frac{k^{2} X}{Y t}\right) \mathcal{G}\left(\frac{1}{2}, 2 k^{2}\right) \mathrm{d} t \\
\sum_{k=1}^{\infty} \tilde{f}\left(\frac{1}{2}, 2 k^{2}\right) \mathcal{G}^{\prime}\left(\frac{1}{2}, 2 k^{2}\right) & =\int_{0}^{\infty} \frac{\Phi(t)}{\sqrt{t}} \sum_{k=1}^{\infty} \widetilde{W}\left(\frac{k^{2} X}{Y t}\right) \mathcal{G}^{\prime}\left(\frac{1}{2}, 2 k^{2}\right) \mathrm{d} t .
\end{aligned}
$$


We then apply (2.3) with $j=1$ for $|k| \geq \sqrt{U Y / X}$ and (2.4) for smaller $k$ to see that

$$
\begin{aligned}
& \sum_{k=1}^{\infty} \widetilde{W}\left(\frac{k^{2} X}{Y t}\right) \mathcal{G}\left(\frac{1}{2}, 2 k^{2}\right)=\frac{Y t}{2 \pi X} \sum_{k=1}^{\infty} \frac{\mathcal{G}\left(\frac{1}{2}, 2 k^{2}\right)}{k^{2}}\left(1-\cos \left(\frac{2 \pi k^{2} X}{Y t}\right)+\sin \left(\frac{2 \pi k^{2} X}{Y t}\right)\right)+O\left(U^{-(1-\epsilon) / 2}\left(\frac{Y}{X}\right)^{(1+\epsilon) / 2}\right), \\
& \sum_{k=1}^{\infty} \widetilde{W}\left(\frac{k^{2} X}{Y t}\right) \mathcal{G}^{\prime}\left(\frac{1}{2}, 2 k^{2}\right)=\frac{Y t}{2 \pi X} \sum_{k=1}^{\infty} \frac{\mathcal{G}^{\prime}\left(\frac{1}{2}, 2 k^{2}\right)}{k^{2}}\left(1-\cos \left(\frac{2 \pi k^{2} X}{Y t}\right)+\sin \left(\frac{2 \pi k^{2} X}{Y t}\right)\right)+O\left(U^{-(1-\epsilon) / 2}\left(\frac{Y}{X}\right)^{(1+\epsilon) / 2}\right) .
\end{aligned}
$$

Applying (3.18) and (3.19) to (3.17), we deduce that

$$
M_{1,2}=X^{3 / 2}(\log X+2 \gamma) C_{1}\left(\frac{Y}{X}\right)+X^{3 / 2} C_{2}\left(\frac{Y}{X}\right)+O\left(X Y^{1 / 2} \log Y U^{-(1-\epsilon) / 2}\left(\frac{Y}{X}\right)^{(1+\epsilon) / 2}+\frac{Y^{3 / 2} \log Y}{U}\right),
$$

where we define for $\alpha \geq 0$,

$$
\begin{aligned}
C_{1}(\alpha)= & \frac{1}{2 \pi} \sum_{k=1}^{\infty} \frac{\mathcal{G}\left(\frac{1}{2}, 2 k^{2}\right)}{k^{2}} \int_{0}^{\alpha} \sqrt{y}\left(1-\cos \left(\frac{2 \pi k^{2}}{y}\right)+\sin \left(\frac{2 \pi k^{2}}{y}\right)\right) d y, \\
C_{2}(\alpha)= & \frac{1}{2 \pi} \sum_{k=1}^{\infty} \frac{\mathcal{G}\left(\frac{1}{2}, 2 k^{2}\right)}{k^{2}} \int_{0}^{\alpha} \log y \sqrt{y}\left(1-\cos \left(\frac{2 \pi k^{2}}{y}\right)+\sin \left(\frac{2 \pi k^{2}}{y}\right)\right) d y \\
& +\frac{1}{2 \pi} \sum_{k=1}^{\infty} \frac{\mathcal{G}^{\prime}\left(\frac{1}{2}, 2 k^{2}\right)}{k^{2}} \int_{0}^{\alpha} \sqrt{y}\left(1-\cos \left(\frac{2 \pi k^{2}}{y}\right)+\sin \left(\frac{2 \pi k^{2}}{y}\right)\right) d y .
\end{aligned}
$$

To estimate $R_{2}$, we note that (see [5, Exercise 3, p. 100]) when $\Re(s)=\epsilon$,

$$
L\left(\frac{1}{2}+s, \chi_{k_{1}}\right) \ll(1+|s|)^{1 / 4+\epsilon} .
$$

Using this together with (3.14) by taking $D=2, E=4$ for all $k$ and (3.15), we deduce from (3.16) that

$$
R_{2} \ll X Y^{\epsilon}\left(\frac{Y}{X}\right)^{2} U^{3} \sum_{|k| \geq 1} \frac{|k|^{\varepsilon}}{|k|^{2}} \ll X Y^{\epsilon}\left(\frac{Y}{X}\right)^{2} U^{3} .
$$

We then conclude from (3.20) and 3.22 that

$$
M^{\prime}=X^{3 / 2}(\log X+2 \gamma) C_{1}\left(\frac{Y}{X}\right)+X^{3 / 2} C_{2}\left(\frac{Y}{X}\right)+O\left(X Y^{1 / 2} \log Y U^{-(1-\epsilon) / 2}\left(\frac{Y}{X}\right)^{(1+\epsilon) / 2}+\frac{Y^{3 / 2} \log Y}{U}+X Y^{\epsilon}\left(\frac{Y}{X}\right)^{2} U^{3} .\right.
$$

3.4. Conclusion. Combining (3.3), (3.11) and (3.23), we see that

$$
\begin{aligned}
S(X, Y)= & \frac{X Y^{1 / 2} \log ^{2} Y}{16} \eta(1)+X Y^{1 / 2} P_{1}(\log Y)+X^{3 / 2}(\log X+2 \gamma) C_{1}\left(\frac{Y}{X}\right)+X^{3 / 2} C_{2}\left(\frac{Y}{X}\right) \\
& +O\left(\frac{X Y^{1 / 2} \log ^{2} Y}{U}+X Y^{\frac{1}{4}+\epsilon} U+(X Y)^{\epsilon} \frac{X Y^{1 / 2}+Y X^{1 / 2}}{\sqrt{U}}+X Y^{1 / 2} \log Y U^{-(1-\epsilon) / 2}\left(\frac{Y}{X}\right)^{(1+\epsilon) / 2}\right. \\
& \left.+\frac{Y^{3 / 2} \log Y}{U}+X Y^{\epsilon}\left(\frac{Y}{X}\right)^{2} U^{3}\right) .
\end{aligned}
$$

By taking

$$
U=\left(\frac{X Y^{1 / 2}+Y X^{1 / 2}}{X Y^{\frac{1}{4}}}\right)^{2 / 3}
$$

we derive the expression (1.4) in Theorem 1.1 and this completes the proof.

Acknowledgments. The author is supported by NSFC grant 11871082 . 


\section{REFERENCES}

[1] M. V. Armon, Averages of real character sums, J. Number Theory 77 (1999), no. 2, 209-226.

[2] J. B. Conrey, D. W. Farmer, and K. Soundararajan, Transition mean values of real characters, J. Number Theory 82 (2000), no. 1, 109-120.

[3] H. Davenport, Multiplicative Number Theory, Third edition, Graduate Texts in Mathematics, vol. 74, Springer-Verlag, Berlin, etc., 2000.

[4] D. R. Heath-Brown, A mean value estimate for real character sums, Acta Arith. 72 (1995), no. 3, $235-275$.

[5] H. Iwaniec and E. Kowalski, Analytic Number Theory, American Mathematical Society Colloquium Publications, vol. 53, American Mathematical Society, Providence, 2004.

[6] M. Jutila, On character sums and class numbers, J. Number Theory 5 (1973), 203-214.

$[7]$, On the mean value of $L(1 / 2, \chi)$ for real characters, Analysis 1 (1981), no. 2, 149-161.

[8] H. L. Montgomery and R. C. Vaughan, Multiplicative number theory. I. Classical theory, Cambridge Studies in Advanced Mathematics, vol. 97, Cambridge University Press, Cambridge, 2007.

[9] K. Soundararajan, Nonvanishing of quadratic Dirichlet L-functions at $s=\frac{1}{2}$, Ann. of Math. (2) 152 (2000), no. 2, $447-488$.

Department of Mathematics, School of Mathematics and Systems Science, Beihang University, P. R. China

E-mail address: penggao@buaa.edu.cn 